\title{
Quadratic Interactions in Finite, One-Dimensional, Photonic Band Gap Structures with Deep Gratings: beyond the Undepleted Pump Regime
}

\author{
G. D'Aguanno ${ }^{a, b, *}$, M. Centini ${ }^{a, b}$, M. Scalora ${ }^{b}$, C. Sibilia $^{a}$, \\ M. Bertolotti ${ }^{a}$, Ch.M. Bowden ${ }^{b}$ And M.J. Bloemer ${ }^{b}$ \\ aINFM at Dipartimento di Energetica, Università di Roma "La Sapienza" \\ via A. Scarpa 16, 00161 Rome, Italy \\ ${ }^{b}$ Weapons Sciences Directorate, Research Development and Engineering Center \\ U.S. Army Aviation \& Missile Command, Building 7804, Redstone Arsenal
}

AL 35898-5000, USA

\begin{abstract}
We theoretically study quadratic interactions in finite, one-dimensional, photonic band gap structures with deep gratings, under strong pumping and global phase matching conditions. For second harmonic generation, we find that above a certain input intensity a dynamics reminiscent of a competitive, multi-wave mixing process takes hold: the pump field is mostly reflected, revealing a novel type of optical limiting behavior, while forward and backward generation is generally balanced. We also study the case of parametric down-conversion, where an intense second harmonic signal is injected in order to control a much weaker fundamental beam. Our results reveal the onset of a new process that has no counterpart in bulk materials: both transmission and reflection display an unexpected, unusual, resonance-like effect as functions of input second harmonic power.
\end{abstract}

PACS numbers: $42.65 . \mathrm{Ky}$

\section{Introduction}

For more than half century semiconductors have had a prominent role to play in almost every field of technology thanks to the ability to tailor their con-

*corresponding author; e-mail: giuseppe.daguanno@uniroma1.it 
ductive properties. Just as tailoring the properties of semiconductors constituted a challenge several decades ago in solid state physics, today tailoring the properties of photonic crystals (PC) may hold the key to achieving significant technological advances in the field of photonics. For this purpose we believe that a new class of materials, called photonic band gap (PBG) structures, appears to hold much promise. One-dimensional (1-D) PBG structures are made by arranging macroscopic dielectric and/or metallic unit cells into a periodic or quasi-periodic array, in order to affect the properties of the light in almost the same way that semiconductor crystals affect the properties of electrons. The periodic arrangement results in allowed and forbidden frequency bands and gaps for the light, in analogy to energy bands and gaps of semiconductors.

The study of PBG materials as such began with the works of Yablonovitch [1] and John [2] on spontaneous emission control and light localization. These contributions gave way to an intense theoretical and experimental investigation of PBG structures that has continued since. Some of the applications that have been proposed over the years include photonic crystals fibers [3], photonic crystals circuits [4], transparent metal-dielectric stacks [5], highly efficient micron-sized devices for nonlinear frequency conversion [6-8]. An up to date review of recent advancements in the field of PBG structures may be found in Ref. [9].

Although the number of experimental and theoretical reports on quadratic interactions in PBG structures is significant [6-8, 10-19], in our view the chapter on quadratic interactions in PBG structures is still far from being considered closed. For instance, the dynamics that takes place under strong pumping conditions, or under conditions of pump depletion, leads to highly unusual and previously unknown effects such as a novel $\chi^{(2)}$-based optical limiting that we will discuss below.

The paper is organized as follows: in Sec. 2 we summarize a generalized coupled mode theory, under the monochromatic approximation, that is valid for arbitrary index modulation and profile, and in the depleted pump regime [20]. In Sec. 3 we numerically integrate the coupled mode equations derived in Sec. 2 using a shooting procedure [21], and study two different regimes characterized by strong pumping conditions: (a) second harmonic generation (SHG), and (b) parametric down-conversion.

\section{The model}

The scalar nonlinear Helmholtz equations governing the quadratic interactions of two linearly polarized plane waves at fundamental frequency (FF) $\omega$, and SH frequency $2 \omega$ in a layered, 1-D, finite structure can be written as [14]:

$$
\frac{\mathrm{d}^{2} E_{\omega}}{\mathrm{d} z^{2}}+\frac{\omega^{2} \varepsilon_{\omega}(z)}{c^{2}} E_{\omega}=-2 \frac{\omega^{2}}{c^{2}} d^{(2)}(z) E_{\omega}^{*} E_{2 \omega},
$$




$$
\frac{\mathrm{d}^{2} E_{2 \omega}}{\mathrm{d} z^{2}}+\frac{4 \omega^{2} \varepsilon_{2 \omega}(z)}{c^{2}} E_{2 \omega}=-4 \frac{\omega^{2}}{c^{2}} d^{(2)}(z) E_{\omega}^{2},
$$

$\varepsilon_{j \omega}(z)(j=1,2)$ are the spatially dependent, linear dielectric functions for the $\mathrm{FF}$ and $\mathrm{SH}$ fields. In general, $\varepsilon_{j \omega}(z)$ are assumed to be complex functions. The condition $\varepsilon_{\omega}(z) \neq \varepsilon_{2 \omega}(z)$ takes into account possible material dispersion. Finally, $d^{(2)}(z)$ is the spatially dependent quadratic coupling function.

In spite of their apparent simplicity, Eqs. (1) admit no known, general analytical solutions. Equations (1) can be integrated numerically by resorting to a nonlinear matrix transfer technique [22], assuming no pump depletion and a weak nonlinearity. Modifications of the matrix transfer technique have been performed to take pump depletion into account [23, 24]. However, in our view those techniques might become in some cases quite cumbersome to implement. The analysis of Eqs. (1) can be simplified considerably by identifying two different spatial scales of variation of the electric fields: (i) a fast-scale, which accounts for oscillations that may occur within a spatial scale on the order of the wavelength due to linear interference effects; and (ii) a slow-scale, which takes into account the nonlinear polarization source terms on the right-hand side of Eqs. (1): the role of the nonlinearity is to modulate the linear solution over a length scale much longer with respect to the fast scale. In order to separate fast and slow-scale variations we introduce a new set of independent variables, $z_{\alpha}=\lambda^{\alpha} z$ with $\alpha=0,1,2 \ldots$, where $\lambda$ is a dimensionless parameter. Once the multiple scales expansion [14, 25-27] has been performed, the procedure calls for the application of the limit $\lambda \rightarrow 1$ to restore the original space variable $z$. The derivative operator is expanded according to the new set of variables, namely:

$$
\frac{\mathrm{d}}{\mathrm{d} z}=\frac{\partial}{\partial z_{0}}+\lambda \frac{\partial}{\partial z_{1}}+\lambda^{2} \frac{\partial}{\partial z_{2}}+\ldots
$$

The linear and nonlinear dielectric functions, $\varepsilon_{j \omega}(z)$ and $d^{(2)}(z)$, respectively, will be considered functions of the fast variable $z_{0}$. The complex field amplitude functions, which we introduce below, will be functions of the slowly varying variables $z_{1}, z_{2}$, etc. The electric fields are also expanded in powers of the perturbing parameter $\lambda$ in a self-consistent manner:

$$
E_{j \omega}=\lambda E_{j \omega}^{(1)}\left(z_{0}, z_{1}, z_{2}, \ldots\right)+\lambda^{2} E_{j \omega}^{(2)}\left(z_{0}, z_{1}, z_{2}, \ldots\right)+\ldots, j=1,2 .
$$

Substituting Eqs. (2-3) into Eqs. (1a-b), and collecting the terms proportional to $\lambda$ we find that the first order expansion of the electric fields can be expressed as follows:

$$
E_{j \omega}^{(1)}=A_{j \omega}^{(+)}\left(z_{1}, z_{2}, \ldots\right) \Phi_{j \omega}^{(+)}\left(z_{0}\right)+A_{j \omega}^{(-)}\left(z_{1}, z_{2}, \ldots\right) \Phi_{j \omega}^{(-)}\left(z_{0}\right)
$$

where $\left\{\Phi_{j \omega}^{( \pm)}\right\}$are the left-to-right (LTR) and right-to-left (RTL) linear modes that are functions of fast variable $z_{0}$. LTR and RTL modes can be calculated using a standard linear matrix transfer technique, assuming a unitary electric field is incident on the structure from LTR for the $\Phi_{j \omega}^{(+)}$modes, and from RTL 
for $\Phi_{j \omega}^{(-)}$the modes [28]. We note that $\Phi_{j \omega}^{( \pm)}$carry information about the linear localization properties of electric field inside the structure, and $A_{j \omega}^{( \pm)}\left(z_{1}, z_{2}, \ldots\right)$ are the complex amplitudes of the fields that depend on the slow variables $z_{1}, z_{2}, \ldots$ In case there are no nonlinear interactions, $A_{j \omega}^{( \pm)}\left(z_{1}, z_{2}, \ldots\right)$ represent constant amplitudes of LTR and RTL incident fields. Then, (a) collecting terms proportional to $\lambda^{2}$; (b) projecting the resulting equations over the LTR and RTL modes using the standard metric $\langle f|g|\rangle \equiv(1 / L) \int_{0}^{L} f^{*}(z) g(z) \mathrm{d} z$; and (c) taking the limit $\lambda \rightarrow 1$, we arrive at four coupled, nonlinear differential equations:

$$
\begin{aligned}
& \sum_{l=+,-} p_{\omega}^{(+, l)} \frac{\mathrm{d} A_{\omega}^{(l)}}{\mathrm{d} z}=\mathrm{i} \frac{\omega}{c} \sum_{(k, l)=(+,-)} \Gamma_{(\omega,+)}^{(k, l)} A_{2 \omega}^{(k)} A_{\omega}^{(l) *}, \\
& \sum_{l=+,-} p_{\omega}^{(-, l)} \frac{\mathrm{d} A_{\omega}^{(l)}}{\mathrm{d} z}=\mathrm{i} \frac{\omega}{c} \sum_{(k, l)=(+,-)} \Gamma_{(\omega,-)}^{(k, l)} A_{2 \omega}^{(k)} A_{\omega}^{(l) *}, \\
& \sum_{l=+,-} p_{2 \omega}^{(+, l)} \frac{\mathrm{d} A_{2 \omega}^{(l)}}{\mathrm{d} z}=\mathrm{i} \frac{\omega}{c} \sum_{(k, l)=(+,-)} \Gamma_{(2 \omega,+)}^{(k, l)} A_{\omega}^{(k)} A_{\omega}^{(l)}, \\
& \sum_{l=+,-} p_{2 \omega}^{(-, l)} \frac{\mathrm{d} A_{2 \omega}^{(l)}}{\mathrm{d} z}=\mathrm{i} \frac{\omega}{c} \sum_{(k, l)=(+,-)} \Gamma_{(2 \omega,-)}^{(k, l)} A_{\omega}^{(k)} A_{\omega}^{(l)},
\end{aligned}
$$

where $p_{j \omega}^{(k, l)}=\left\langle\Phi_{j \omega}^{(k)} \mid \hat{p}_{j \omega} \Phi_{j \omega}^{(l)}\right\rangle$, for $j=1,2$ and $k, l=+,-, \quad \Gamma_{(\omega, n)}^{(k, l)}=$ $\left\langle\Phi_{\omega}^{(n)} \mid d^{(2)} \Phi_{2 \omega}^{(k)} \Phi_{\omega}^{(l) *}\right\rangle, \Gamma_{(2 \omega, n)}^{(k, l)}=\left\langle\Phi_{2 \omega}^{(n)} \mid d^{(2)} \Phi_{\omega}^{(k)} \Phi_{\omega}^{(l)}\right\rangle$, for $n, k, l=+,-$. The $p_{j \omega}^{(k, l)}$ are matrix elements of the momentum operator $\hat{p}_{j \omega} \equiv-\mathrm{i}(c / j \omega) \mathrm{d} / \mathrm{d} z$ calculated over the RTL and LTR linear modes.

The overlap coefficients $\Gamma_{(j \omega, n)}^{(k, l)}$ are effective, complex coupling coefficients that reflect the way in which the LTR and RTL modes sample the distribution of the nonlinearity $d^{(2)}(z)$ over the structure. The values of $\Gamma_{(j \omega, n)}^{(k, l)}$ are maximized, and can be greater than the magnitude of $d^{(2)}(z)$, when the fields interact coherently inside the structure. Since no assumptions were made regarding the type of grating, Eqs. (5) are valid for arbitrary index profiles and tuning conditions. We note that this formulation allows analytical solutions in the undepleted pump regime [20]. We also note that Eqs. (5) are valid when the steady state regime is approached. In general, the steady state regime is approached when the interaction time (dwell time) of an input pulse in the PBG structure is much shorter than the duration of the pulse itself. In the case of transform limited pulses, this means that the spectral bandwidth of the pulse should be much narrower than the spectral bandwidth of each transmission resonance. In the PBG structure we consider (see Fig. 1), the maximum interaction time $\tau_{\text {int }} \approx 1 / \Delta \omega_{\text {resonance }}$ at the band edge resonance is approximately 1 ps. This means that for all intents and purposes the dynamics of input pulses of a few tens of picoseconds can be considered to be in the steady state regime. 
Equations (5) may be recast in a simpler form, provided we restrict our attention to the case when both the $\mathrm{FF}$ and $\mathrm{SH}$ modes are tuned to transmission resonances, and the structure is not absorptive. We choose these tuning conditions because off-resonant interactions become quite inefficient for at least two reasons: (a) impossibility to find phase matching conditions, and (b) poor field localization inside the structure [8, 10, 13]. Therefore, if resonant tuning conditions restrictions are imposed, the diagonal matrix elements of the momentum operator can be calculated analytically: $p_{j \omega}^{(+,+)}=\left\langle\Phi_{j \omega}^{(+)} \mid \hat{p}_{j \omega} \Phi_{j \omega}^{(+)}\right\rangle=1, p_{j \omega}^{(-,-)}=$ $\left\langle\Phi_{j \omega}^{(-)} \mid \hat{p}_{j \omega} \Phi_{j \omega}^{(-)}\right\rangle=-1$.

Further simplifications can be performed by considering symmetric or periodic structures. The off-diagonal elements of the momentum operator can be estimated as $p_{j \omega}^{(-,+)} \approx-\left[p_{j \omega}^{(+,-)}\right] \approx(\lambda / 2 \pi j L) \sin \left[\phi_{\mathrm{t}}(j \omega)\right]$ [20], where $L$ is the total length of the structure, and $\phi_{\mathrm{t}}(j \omega)$ is the phase of its linear transmission function, $t_{j \omega}=\sqrt{T_{j \omega}} \exp \left[\mathrm{i} \phi_{\mathrm{t}}(j \omega)\right]$. For periodic structures under resonant conditions, the phase of the transmitted field is proportional to multiples of $\pi[29,30]$. Therefore, the off-diagonal elements of the momentum operator become negligible with respect to the diagonal ones. For symmetric PBG structures, the off-diagonal elements are also negligible, provided $\lambda \ll 2 \pi L$ ( $\lambda$ is the wavelength of the FF field in vacuum). Simply stated, this amounts to choosing a structure that is at least several wavelengths long. Finally, neglecting the off-diagonal elements with respect to the diagonal ones, the coupled mode equations can be rewritten in the following simplified form:

$$
\begin{aligned}
& \frac{\mathrm{d} A_{\omega}^{(+)}}{\mathrm{d} z}=\mathrm{i} \frac{\omega}{c} \sum_{(k, l)=(+,-)} \Gamma_{(\omega,+)}^{(k, l)} A_{2 \omega}^{(k)} A_{\omega}^{(l) *}, \\
& \frac{\mathrm{d} A_{\omega}^{(-)}}{\mathrm{d} z}=-\mathrm{i} \frac{\omega}{c} \sum_{(k, l)=(+,-)} \Gamma_{(\omega,-)}^{(k, l)} A_{2 \omega}^{(k)} A_{\omega}^{(l) *}, \\
& \frac{\mathrm{d} A_{2 \omega}^{(+)}}{\mathrm{d} z}=\mathrm{i} \frac{\omega}{c} \sum_{(k, l)=(+,-)} \Gamma_{(2 \omega,+)}^{(k, l)} A_{\omega}^{(k)} A_{\omega}^{(l)}, \\
& \frac{\mathrm{d} A_{2 \omega}^{(-)}}{\mathrm{d} z}=-\mathrm{i} \frac{\omega}{c} \sum_{(k, l)=(+,-)} \Gamma_{(2 \omega,-)}^{(k, l)} A_{\omega}^{(k)} A_{\omega}^{(l)} .
\end{aligned}
$$

We stress that if one wishes to investigate structures such that the fields are not resonant or absorptive, one should resort Eqs. (5), which retain their general validity in the steady state regime. Analysis of deep grating and model equations in quadratically nonlinear periodic media has been reported for the first time in Ref. [31, 32], where the field is expanded in term of Bloch modes according to the approach used by de Sterke and Sipe [26] for cubically nonlinear periodic media. We indeed pursue solutions based on LTR and RTL linear modes of the structure that explicitly take into account the boundary conditions at the input and 
the output interfaces, as explained at length in Ref. [20]. Once again, we stress that our approach is valid for any kind of index modulation (periodic or not).

\section{Results and discussion}

$$
\text { 3.1. } S H G
$$

As an example, let us consider a PBG structure composed of 59 alternating layers of air (index unity at all frequencies) and an ideal, generic, quadratic dielectric material. The index of refraction of the material at $\lambda=1.55 \mu \mathrm{m}$ is $n_{1}(\omega)=3.342$, and its index of refraction at the SH frequency is $n_{1}(2 \omega)=3.61$; the nonlinear coefficient is assumed to be $d^{(2)}=120 \mathrm{pm} / \mathrm{V}^{\dagger}$. The structure under consideration is symmetric, and more details are described in the caption of Fig. 1. The FF field is tuned at the first transmission resonance near the first order band gap, and the SH field is tuned at the second transmission resonance near the second order band gap (see Fig. 1). Tuning in this fashion, the SH field is globally phase-matched with the FF field [13].

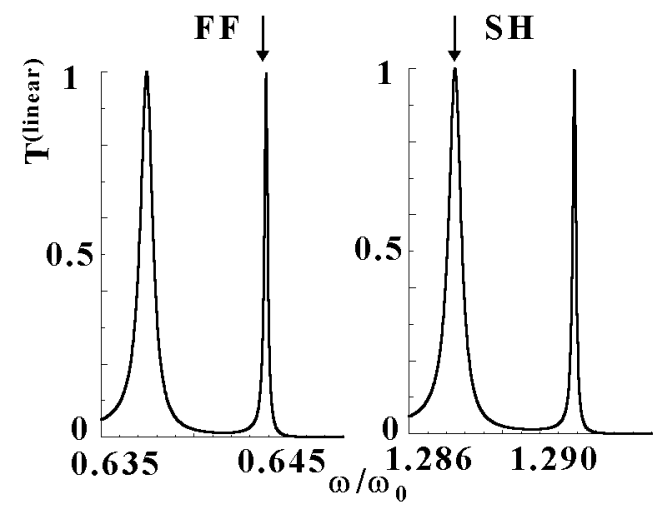

Fig. 1. Linear transmittance vs. normalized frequency $\omega / \omega_{0}, \omega_{0}=2 \pi c / \lambda_{0}, \lambda_{0}=1 \mu \mathrm{m}$. The structure is composed of 59 alternating layers of air and a dielectric material. The index of refraction of the dielectric material at FF $(\lambda=1.55 \mu \mathrm{m})$ is $n_{1}(\omega)=3.342$ and its index of refraction at the SH frequency is $n_{1}(2 \omega)=3.61$. The layers have thicknesses $a=90 \mathrm{~nm}$ (air) and $b=150 \mathrm{~nm}$ (dielectric material), the total length of the structure is $L=7.11 \mu \mathrm{m}$. The arrow identifies the tuning of the $\mathrm{FF}$ and $\mathrm{SH}$ field, respectively.

We have numerically integrated Eqs. (6) $)^{\ddagger}$ using a shooting procedure [21]. In Fig. 2 we show the reflected and transmitted FF field, the backward and forward

${ }^{\dagger}$ Here we refer to an ideal, generic, quadratic material with a relatively high nonlinearity, and ignore two-photon absorption and Kerr effect. These effects might become important under strong pumping conditions, as might be the case in AlGaAs, for example. The study of competing nonlinearities is beyond the scope of the present work.

†For the PBG structure we consider the off-diagonal elements of the momentum operator which are approximately two or three orders of magnitude smaller than the diagonal ones. Henceforth, we carry out the numerical integrations of Eqs. (6) instead of Eqs. (5) 


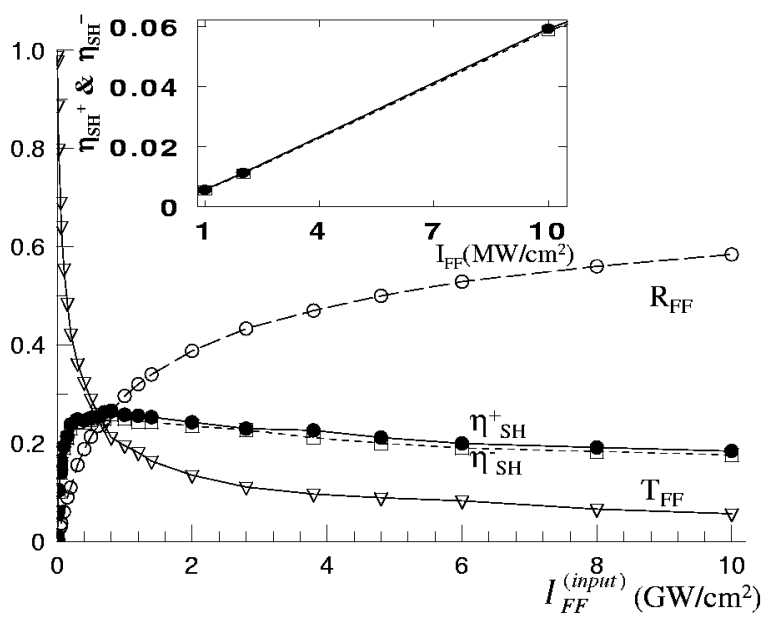

Fig. 2. Forward SH conversion efficiency $\eta_{S \mathrm{H}}^{+}=\left|A_{2 \omega}^{(+)}(L)\right|^{2} /\left|A_{\omega}^{(+)}(0)\right|^{2}$ (filled circle-solid line), backward SH conversion efficiency $\eta_{S H}^{-}=\left|A_{2 \omega}^{(-)}(0)\right|^{2} /\left|A_{\omega}^{(+)}(0)\right|^{2}$ (open squared-short dashed line), reflected FF field $R_{\mathrm{FF}}=\left|A_{\omega}^{(-)}(0)\right|^{2} /\left|A_{\omega}^{(+)}(0)\right|^{2}$ (open circle-long dashed line), and transmitted FF field $T_{\mathrm{FF}}=\left|A_{\omega}^{(+)}(L)\right|^{2} /\left|A_{\omega}^{(+)}(0)\right|^{2}$ (open triangle-solid line) vs. input FF intensity $I_{\mathrm{FF}}^{\text {input }}=(1 / 2) \varepsilon_{0} c\left|A_{\omega}^{(+)}(0)\right|^{2}$. The FF intensity is incident from vacuo on the PBG structure in the LTR direction. The nonlinear coefficient of the dielectric material is $d^{(2)}=120 \mathrm{pm} / \mathrm{V}$. The symbols (circles, triangles, and squares) represent the values calculated by numerically integrating Eqs. (6) via a shooting procedure. Note that the energy among the four channels is conserved, as one might expect: $R_{\mathrm{FF}}+T_{\mathrm{FF}}+\eta_{\mathrm{SH}}^{+}+\eta_{\mathrm{SH}}^{-}=1$. Inset: Magnification of the forward and backward SH conversion efficiencies in the region of negligible depletion of the FF field.

SH conversion efficiencies as a function of the intensity of the incident $\mathrm{FF}$ field. The following are, in our view, the most salient points of Fig. 2:

(i) The forward and backward SH conversion efficiencies are approximately the same at all times. This is a consequence of the fact that the structure has strong feedback due to the high-index contrast between layers. Choosing smaller index contrasts between adjacent layers causes an imbalance between forward and backward generation. In the limiting case where there is no index mismatch (i.e., bulk) SHG occurs entirely in the forward direction.

(ii) Even under global phase matching conditions, SH conversion efficiency does not increase monotonically to deplete the pump, as one might be tempted to think since that is how bulk materials behave. We calculate a total maximum conversion efficiency of roughly $50 \%$, equally distributed between the forward and the backward SH channels, for input FF intensities of approximately $0.6 \mathrm{GW} / \mathrm{cm}^{2}$. The total conversion efficiency then decreases to about $40 \%$ for input FF intensities of $\sim 10 \mathrm{GW} / \mathrm{cm}^{2}$. This behavior is unusual if one thinks of a phase-matched 
process and neglects the various components, or channels, that compete for the same available energy.

(iii) In fact, the forward FF channel is not only converting energy into SH forward and backward channels, but it is also strongly coupling to the backward FF channel, leading to excess reflections. This result is new and unexpected. Therefore, the process of SHG in a PBG structure under conditions of pump depletion should be more appropriately regarded as a multi-wave mixing process. Quite surprisingly, the process that is privileged under strong pumping conditions is not the SH generation; in fact, most of the energy is converted from the FF forward channel to the FF backward channel. The figure suggests that pump reflections exceed $60 \%$, while its transmission drops to approximately $5 \%$.

(iv) The dynamics outlined above is also strongly suggestive of a novel, unusual optical limiting behavior. Using quadratic interactions for optical limiting purposes would result in much faster devices compared with other, more traditional schemes of optical limiters based on cubic nonlinearities. In addition, the device would not suffer from the detrimental effects typical of cubic materials, such as absorption, heating or saturation, since energy is always stored in the field, and not transferred to the material: our proposed optical limiter would act on the transmitted FF (see Fig. 2) by limiting its energy on the basis of a purely quadratic interaction. More specifically, it can be shown that for our structure the output FF intensity scales approximately as follows: $I_{\mathrm{FF}}^{\text {(output) }} \approx a \sqrt{I_{\mathrm{FF}}^{(\text {input })}}$ with $a \cong$ $0.19(\mathrm{GW})^{1 / 2} / \mathrm{cm}$.

The physical mechanism that in this case leads to optical limiting is different than the physical mechanism required for the onset of optical limiting in the case of cubic nonlinearities. In the latter case, optical limiting can occur because of

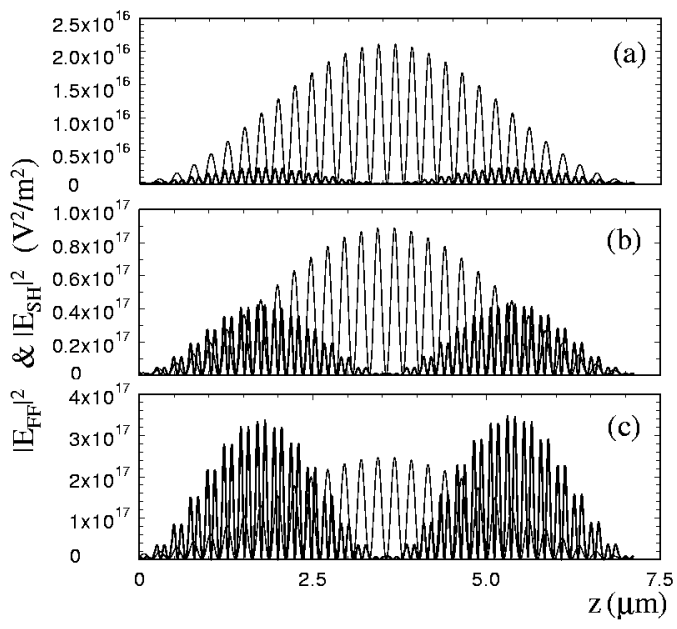

Fig. 3. Absolute value squared of the FF field (thin solid line) and of the SH field (thick solid line) inside the PBG structure, for different values of the input intensity; (a) $I_{\mathrm{FF}}^{(\text {input) }}=0.05 \mathrm{GW} / \mathrm{cm}^{2}$, (b) $I_{\mathrm{FF}}^{(\text {input })}=0.5 \mathrm{GW} / \mathrm{cm}^{2}$, (c) $I_{\mathrm{FF}}^{\text {(input) }}=4.8 \mathrm{GW} / \mathrm{cm}^{2}$. 
dynamical, spectral band-shift in the location of the band gap [33], and/or in combination with nonlinear absorption. In our case, the mechanism of the optical limiting process consists of an energy exchange between the FF forward and the FF backward channels, mediated by the SHG process.

To highlight the different mechanisms that drive optical limiting in the case of quadratic and cubic nonlinearities, in Figs. 3 we show the FF and SH field profiles inside the PBG structure during the SHG process, for different values of the input intensity. The Figures suggest that the FF remains well localized inside the structure, even when its transmission drops to values less than $10 \%$ (Fig. 3c). We do not record any exponentially decaying tails in the $\mathrm{FF}$ mode, which are typical of cubic nonlinearities that cause shifts of the band edge, and push the FF inside the gap [33]. Finally, the dynamics described in Fig. 2 also suggests another application, namely a phase-insensitive, nonlinear reflector. In our case, the reflection coefficient can be approximately described by the following scaling law: $R_{\mathrm{FF}} \approx b \sqrt[3]{T_{\mathrm{FF}}^{\text {(input) }}}$, where $b \cong 0.28\left(\mathrm{~cm}^{2} / \mathrm{GW}\right)^{1 / 3}$. A more detailed analysis of $S H G$ under pump depletion conditions will be presented elsewhere [34].

Limiting effects during SHG have also been discussed in context of the so-called "parametric gap solitons", the reader interested may consult the review article by Trillo et al. [35] and the extended list of references cited therein.

\subsection{Parametric down-conversion}

We now investigate the parametric down-conversion regime that arises when an intense SH field is injected together with a weak fundamental field. The tuning conditions are the same as those outlined in Fig. 1 for the SHG process. When both the input $\mathrm{FF}$ and $\mathrm{SH}$ field are present, the interaction will be dependent on the relative phase difference between the input fields. In Figs. 4 we show the reflected and transmitted $\mathrm{FF}$ fields vs. the relative input phase difference for fixed input FF and SH fields. Taking the SH input intensity to be at least eight orders of magnitude greater than the FF input intensity $\left(I_{\mathrm{SH}}=13.27 \mathrm{MW} / \mathrm{cm}^{2}\right.$ and $I_{\mathrm{FF}}=0.132 \mathrm{~W} / \mathrm{cm}^{2}$ ), our calculations show that the $\mathrm{SH}$ field remains practically undepleted at all times. Figures 4 suggest that transmission and reflection coefficients are periodic functions of $\delta \phi[22]$, as one might expect. In addition, the $\mathrm{FF}$ transmitted and reflected fields undergo an amplification process that is enhanced for $\delta \phi=3 \pi / 4+m \pi$, while de-amplification takes place for $\delta \phi=\pi / 4+m \pi(m=0,1,2 \ldots)$.

In Figs. 5 we show: (a) the reflection and transmission, and (b) the phases of the FF output field vs. the SH input intensity. The phase difference of the input fields is chosen to be $\delta \phi=3 \pi / 4$. The Figures also show a new and interesting phenomenon: the FF field displays an unusual, resonance-like dynamics by increasing reflection and transmission (as a function of input SH intensity) first sharply followed by an equally sharp decrease beginning $\sim 170 \mathrm{MW} / \mathrm{cm}^{2}$. This behavior indicates an inversion of the gain process, which favors energy flow from the FF 


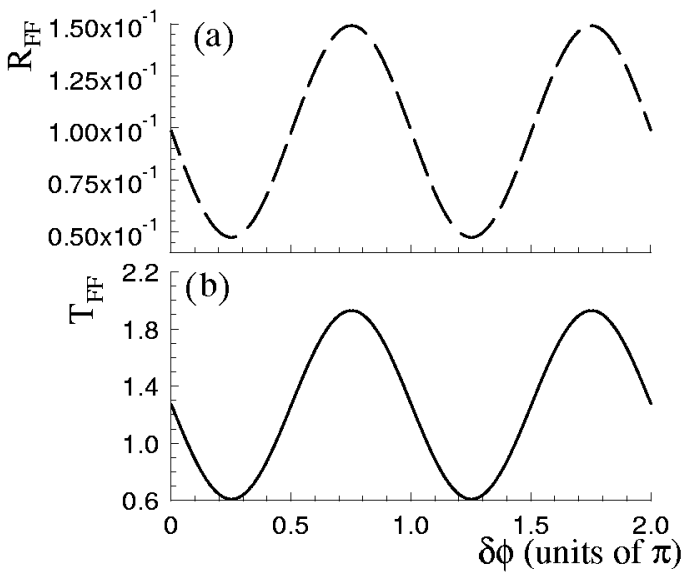

Fig. 4. (a) $R_{\mathrm{FF}}=\left|\mathrm{A}_{\omega}^{(-)}(0)\right|^{2} /\left|\mathrm{A}_{\omega}^{(+)}(0)\right|^{2}$ (dashed line) and (b) $T_{\mathrm{FF}}=$ $\left|\mathrm{A}_{\omega}^{(+)}(\mathrm{L})\right|^{2} /\left|\mathrm{A}_{\omega}^{(+)}(0)\right|^{2}$ (solid line) vs. the phase difference of the input fields $\delta \phi=$ $\phi_{\omega}^{(+)}(0)-\phi_{2 \omega}^{(+)}(0)$. The input fields intensities are respectively $I_{\mathrm{FF}}=1.32 \times 10^{-1} \mathrm{~W} / \mathrm{cm}^{2}$ and $I_{\mathrm{SH}}=13.27 \mathrm{MW} / \mathrm{cm}^{2}$. Note that in our case the amplification process is enhanced for input phase differences around $3 \pi / 4+m \pi$.

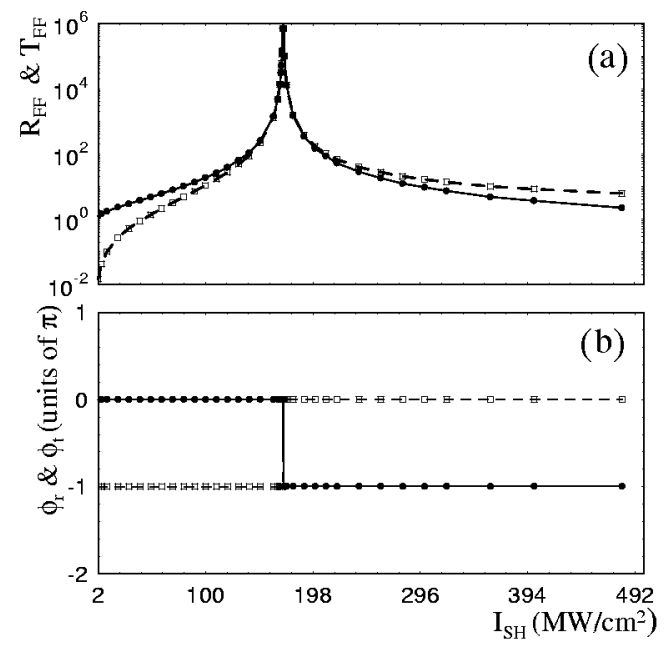

Fig. 5. (a) $R_{\mathrm{FF}}=\left|\mathrm{A}_{\omega}^{(-)}(0)\right|^{2} /\left|\mathrm{A}_{\omega}^{(+)}(0)\right|^{2}$ (open squared-dashed line) and $T_{\mathrm{FF}}=$ $\left|\mathrm{A}_{\omega}^{(+)}(\mathrm{L})\right|^{2} /\left|\mathrm{A}_{\omega}^{(+)}(0)\right|^{2}$ (filled circle-solid line) vs. SH input intensity. (b) Phase of the FF field upon reflection $\phi_{\mathrm{r}}=\arg \left[A_{\omega}^{(-)}(0) / A_{\omega}^{(+)}(0)\right]$ (open squared-dashed line) and phase of the FF field upon transmission $\phi_{\mathrm{t}}=\arg \left[A_{\omega}^{(+)}(L) / A_{\omega}^{(+)}(0)\right]$ (filled circle-solid line) vs. $\mathrm{SH}$ input intensity. The FF input intensity is $I_{\mathrm{FF}}=1.32 \times 10^{-1} \mathrm{~W} / \mathrm{cm}^{2}$ and the phase difference of the input fields is $\delta \phi=3 \pi / 4$. The symbols (circles and squares) represent the values calculated by numerically integrating Eqs. (6) via a shooting procedure. 
back to the SH field. The inversion of gain is accompanied by a $\pi$ phase shift in both reflected and transmitted fields.

While it is not atypical for $\pi$ phase shifts to occur when the inversion of the quadratic process takes place in bulk materials, under perfect phase matching conditions [36-38], we find that $\pi$ phase shifts also occur under resonant conditions in a PBG structures. The reflected and transmitted FF fields reach their maxima $\left(I_{\mathrm{FF}}^{\text {reflected }} \approx I_{\mathrm{FF}}^{\text {transmitted }} \approx 10^{-1} \mathrm{MW} / \mathrm{cm}^{2}\right)$ for $I_{\mathrm{SH}} \sim 170 \mathrm{MW} / \mathrm{cm}^{2}$. Even in this case the SH field remains practically undepleted due to the inversion of gain. This result is also new and unexpected.

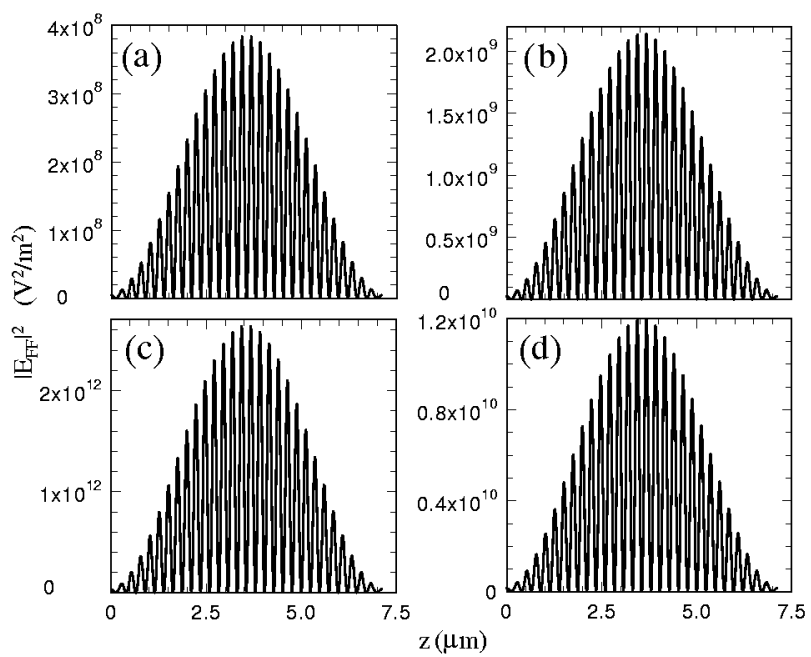

Fig. 6. Absolute value squared of the FF field inside the PBG structure, for different values of the input $\mathrm{SH}$ intensity; (a) $I_{\mathrm{SH}}^{\text {input }}=50 \mathrm{MW} / \mathrm{cm}^{2}$, (b) $I_{\mathrm{SH}}^{\text {input }}=110 \mathrm{MW} / \mathrm{cm}^{2}$, (c) $I_{\mathrm{SH}}^{\text {input }}=169 \mathrm{MW} / \mathrm{cm}^{2}$, (d) $I_{\mathrm{SH}}^{\text {input }}=200 \mathrm{MW} / \mathrm{cm}^{2}$.

In Fig. 6 we show the absolute value squared of the $\mathrm{FF}$ field inside the PBG structure, for different values of the input SH intensity. The Figures suggest that the FF remains well localized inside the structure for SH intensities beyond $I_{\mathrm{SH}} \sim 170 \mathrm{MW} / \mathrm{cm}^{2}$, with tuning conditions that remain consistent with the peak of the first resonance near the band edge. Once again we do not record any exponentially decaying tails in the FF mode, or a push of FF toward the gap. The Figures also suggest another application: an intensity-controlled, true time, optical delay line. As outlined in Ref. [39], the tunneling time of a quasi monochromatic pulse that traverses a finite barrier is directly proportional to the electromagnetic energy density stored within the barrier. Figure 6 thus suggests that the group velocity of the FF pulse may be modulated by controlling SH pumping levels: the pulse is slowed down for intensities below $I_{\mathrm{SH}} \sim 170 \mathrm{MW} / \mathrm{cm}^{2}$, and speeded up above these values. Following Ref. [35], the tunneling time of the transmitted FF 
field can be calculated as follows:

$$
\tau_{\omega}=\frac{1}{2\left|E_{\omega}^{\text {input }}\right|{ }^{2} c} \int_{0}^{L}\left[\varepsilon_{\omega}(z)\left|E_{\omega}\right|^{2}+\frac{c^{2}}{\omega^{2}}\left|\frac{\mathrm{d} E_{\omega}}{\mathrm{d} z}\right|^{2}\right] \mathrm{d} z .
$$

Finally, in Fig. 7 we show the transmitted and reflected FF fields for an input phase difference $\delta \phi=\pi / 4$. We note that the interaction is quite inefficient compared to the case just studied, i.e., Fig. 5. Figure 7 also suggests that the transmitted FF field monotonically decreases, while the FF reflected field monotonically increases: no $\pi$ phase shift occurs to invert energy flow, and the FF tends to be reflected for the most part.

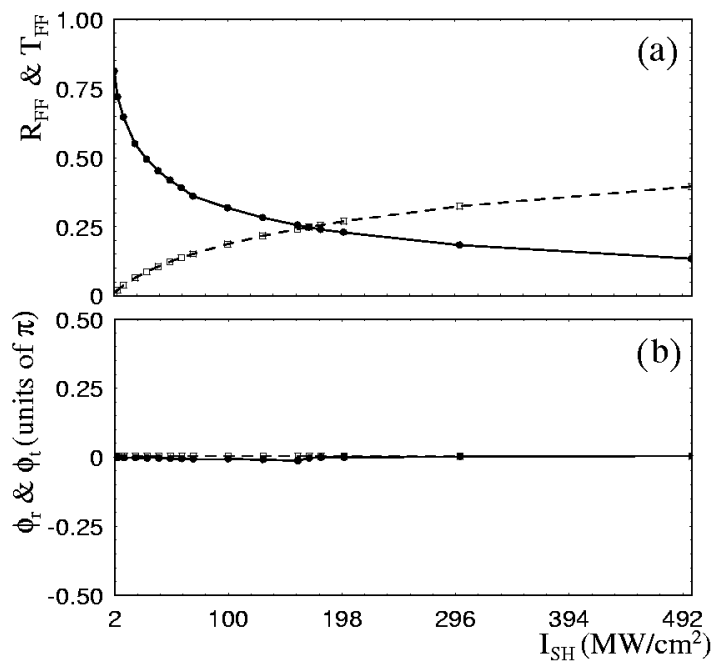

Fig. 7. (a) $R_{\mathrm{FF}}=\left|\mathrm{A}_{\omega}^{(-)}(0)\right|^{2} /\left|\mathrm{A}_{\omega}^{(+)}(0)\right|^{2}$ (open squared-dashed line) and $T_{\mathrm{FF}}=$ $\left|A_{\omega}^{(+)}(0)\right|^{2} /\left|A_{\omega}^{(+)}(0)\right|^{2}$ (filled circle-solid line) vs. SH input intensity. (b) Phase of the FF field upon reflection $\phi_{\mathrm{r}}=\arg \left[A_{\omega}^{(-)}(0) / A_{\omega}^{(+)}(0)\right]$ (open squared-dashed line) and phase of the FF field upon transmission $\phi_{\mathrm{t}}=\arg \left[A_{\omega}^{(+)}(L) / A_{\omega}^{(+)}(0)\right]$ (filled circle-solid line) vs. $\mathrm{SH}$ input intensity. The FF input intensity is $I_{\mathrm{FF}}=1.32 \times 10^{-1} \mathrm{~W} / \mathrm{cm}^{2}$ and the phase difference of the input fields is $\delta \phi=\pi / 4$.

\section{Conclusions}

In summary, we have analyzed the properties of nonlinear quadratic interactions near the photonic band edge under strong pumping and global phase matching conditions. Our results reveal that, if the pump is allowed to deplete, several new phenomena can be identified, including a novel, $\chi^{(2)}$-based optical limiting behavior for SHG, and an unexpected saturation of the up-conversion process. For parametric down-conversion processes, we find that above a certain threshold value an inversion of gain occurs, and the FF field gives energy back to the SH field. Both transmitted and reflected FF field components then display 
sharp resonance-like behavior, which does not allow the pump to become depleted at any time. Nevertheless, this dynamics can lead to a gain-modulated true time delay line.

We thank Nadia Mattiucci for interesting discussions. G. D’Aguanno and M. Centini thank the U.S. Army and the Army Research Laboratory-European Research Office for partial financial support.

\section{References}

[1] E. Yablonovitch, Phys. Rev. Lett. 58, 2059 (1987).

[2] S. John, Phys. Rev. Lett. 58, 2486 (1987).

[3] J.C. Knight, T.A. Birks, P.St.J. Russel, D.M. Atkin, Opt. Lett. 21, 1547 (1996).

[4] J.D. Joannopoulos, P.R. Villeneuve, S.H. Fan, Nature 386, 143 (1997).

[5] M.J. Bloemerm, M. Scalora, Appl. Phys. Lett. 72, 1676 (1998).

[6] M. Scalora, M.J. Bloemer, C.M. Bowden, G. D'Aguanno, M. Centini, C. Sibilia, M. Bertolotti, Y. Dumeige, I. Sagnes, P. Vidakovic, A. Levenson, Opt. $\because$ Photon. News 12, 36 (2001).

[7] Y. Dumeige, P. Vidakovic, S. Sauvage, I. Sagnes, J.A. Levenson, C. Sibilia, M. Centini, G. D'Aguanno, M. Scalora, Appl. Phys. Lett. 78, 3021 (2001).

[8] G. D'Aguanno, M. Centini, M. Scalora, C. Sibilia, Y. Dumeige, P. Vidakovic, J.A. Levenson, M.J. Bloemer, C.M. Bowden, J.W. Haus, M. Bertolotti, Phys. Rev. E 64, 16609 (2001).

[9] Nanoscale Linear and Nonlinear Optics, Eds. M. Bertolotti, C.M. Bowden, C. Sibilia, AIP Conf. Proc., Vol. 560, AIP, New York 2001.

[10] M. Scalora, M.J. Bloemer, A.S. Manka, J.P. Dowling, C.M. Bowden, R. Viswanathan, J.W. Haus, Phys. Rev. A 56, 3166 (1997).

[11] C. Cojocaru, J. Martorell, R. Villaseca, E. Fazio, J. Trull, Appl. Phys. Lett. 74, 504 (1999).

[12] A.V. Balakin, D. Boucher, V.A. Bushev, N.I. Koroteev, B.I. Mantsyzov, P. Masselin, I.A. Ozheredov, A.P. Shurinov, Opt. Lett. 24, 793 (1999).

[13] M. Centini, C. Sibilia, M. Scalora, G. D'Aguanno, M. Bertolotti, M. Bloemer, C.M. Bowden, I. Nefedov, Phys. Rev. E 60, 4891 (1999).

[14] G. D'Aguanno, M. Centini, C. Sibilia, M. Bertolotti, M. Scalora, M. Bloemer, C.M. Bowden, Opt. Lett. 24, 1663 (1999).

[15] S. Saltiel, Y.S. Kivshar, Opt. Lett. 25, 1204 (2000).

[16] A.M. Zheltikov, A.V. Tarasishin, S.A. Magnitski, JETP 91, 298 (2000).

[17] G. D’Aguanno, E. Angelillo, C. Sibilia, M. Scalora, M. Bertolotti, J. Opt. Soc. Am. B 17, $1188(2000)$.

[18] C. De Angelis, F. Gringoli, M. Midrio, D. Modotto, J.S. Aitchison, G.F. Nalesso, J. Opt. Soc. Am. B 18, 348 (2001).

[19] M. Centini, G. D’Aguanno, M. Scalora, C. Sibilia, M. Bertolotti, M.J. Bloemer, C.M. Bowden, Phys. Rev. E 64, 46606 (2001). 
[20] G. D’Aguanno, M. Centini, M. Scalora, C. Sibilia, M. Bertolotti, M.J. Bloemer, C.M. Bowden, J. Opt. Soc. Am. B 19, 2111 (2002) and references therein.

[21] W.H. Press, B.P. Flannery, S.A. Teukolsky, W.T. Vetterling, Numerical Recipies in C, Cambridge University Press, Cambridge 1988.

[22] M. Centini, C. Sibilia, G. D’Aguanno, M. Scalora, M.J. Bloemer, C.M. Bowden, M. Bertolotti, Opt. Commun. 184, 283 (2000).

[23] D.S. Bethune, J. Opt. Soc. Am. B 6, 910 (1995).

[24] N. Hashizume, M. Ohashi, T. Kondo, R. Ito, J. Opt. Soc. Am. B 12, 1894 (1995).

[25] A. Nayfeh, John Wiley and Sons, New York 1993.

[26] C.M. de Sterke, J.E. Sipe, Phys. Rev. A 38, 5149 (1988).

[27] J.W. Haus, R. Viswanathan, M. Scalora, A.G. Kalocsai, J.D. Cole, J. Theimer, Phys. Rev. A 57, 2120 (1998).

[28] O. Di Stefano, S. Savasta, R. Girlanda, J. Mod. Opt. 48, 67 (2001).

[29] P. Yeh, Optical Waves in Layered Media, John Wiley and Sons, New York 1988.

[30] J.M. Bendickson, J.P. Dowling, M. Scalora, Phys. Rev E 53, 4107 (1996).

[31] A. Arraf, C.M. de Sterke, Phys. Rev. E 58, 7951 (1998) and references therein.

[32] C. Conti, G. Assanto, S. Trillo, Opt. Expr. 3, 389 (1998) and refrences therein.

[33] M. Scalora, J.P. Dowling, C.M. Bowden, M.J. Bloemer, Phys. Rev. Lett. 73, 1368 (1994)

[34] G. D’Aguanno, M. Centini, M. Scalora, C. Sibilia, M. Bertolotti, M.J. Bloemer, C.M. Bowden, to appear in Phys. Rev. E (2003).

[35] S. Trillo, C. Conti, G. Assanto, A.V. Buryak, Chaos 10, 590 (2000) and references therein.

[36] A.L. Belostosky, A.S. Leonov, A.V. Maleshko, Opt. Lett. 19, 856 (1994).

[37] G. D'Aguanno, C. Sibilia, E. Fazio, M. Bertolotti, Opt. Commun. 142, 75 (1997).

[38] G. D’Aguanno, C. Sibilia, E. Fazio, E. Ferrari, M. Bertolotti, J. Mod. Opt. 45, 1049 (1998).

[39] G. D’Aguanno, M. Centini, M. Scalora, C. Sibilia, M.J. Bloemer, C.M. Bowden, J.W. Haus, M. Bertolotti, Phys. Rev. E 63, 36610 (2001). 